\title{
POLYNOMIAL DOMINANTS
}

PAUL CIVIN

Let $f(x)$ be a continuous function of period $2 \pi$, and let $F_{n}(x)$ be a trigonometric polynomial of degree $n$ which dominates $f(x)$ at the points $x_{i}{ }^{n}=2 j \pi /(2 n+1)$ where $j=0, \cdots, 2 n$, that is, $F_{n}\left(x_{j}{ }^{n}\right) \geqq\left|f\left(x_{j}{ }^{n}\right)\right|$. The present paper is concerned with relations of inequality between various integral means of the functions $f(x)$ and $F_{n}(x)$. A similar problem was considered by Hardy and Littlewood [2] ${ }^{1}$ under the hypothesis that the Fourier series of a function $F(x)$ was a majorant of the Fourier series of $f(x)$. A further variation concerning the means of the Fourier coefficients under the hypothesis of functional dominance, that is, $F(x) \geqq|f(x)|$, was treated by the author [1]. In the present work, heavy reliance is made on the results of Marcinkiewicz and Zygmund in their paper Mean values of trigonometric polynomials [4]. This paper will be referred to in the sequel as MV.

The results which are obtained are of the type

$$
M_{a}(f) \leqq A M_{b}\left(F_{n}\right)+B \omega(2 \pi / n),
$$

where (i) $M_{\alpha}(g)$ represents some integral mean of $g(x)$; (ii) $A$ and $B$ are constants independent of $n$ and the functions $f(x)$ and $F_{n}(x)$, but dependent on the particular mean $M_{a}$; and (iii) $\omega(\delta)$ is the modulus of continuity of $f(x)$.

It can easily be seen that neither term on the right of inequality (1) can be omitted. Suppose first that, for some $n, f\left(x_{j}{ }^{n}\right)=0$ but $f(x)$ is not identically zero, and also that $F_{n}\left(x_{j}^{n}\right)=0$ so that $F_{n}(x)=0$. Then the left side of (1) will not be zero while the first term on the right vanishes, and consequently the second term on the right is essential. Finally, if the first term were not present in the right member of (1), the left side would be different from zero for non-null functions while the right side would tend to zero as $n \rightarrow \infty$.

Throughout the remainder of the paper we shall suppose that $x_{j}^{n}=2 j \pi /(2 n+1)$; that $\phi_{2 n+1}(t)=x_{j}$ when $x_{j} \leqq t<x_{j+1}$; and that a letter $A, B, \cdots$ with subscript will denote a constant depending only on those parameters appearing in the subscript. The same letter may have a different value at each appearance.

THEOREM 1. For $1<r<\infty$,

Presented to the Society, February 26, 1946; received by the editors December 26, 1945.

${ }^{1}$ Numbers in brackets refer to the references cited at the end of the paper. 
(2) $\left(\int_{0}^{2 \pi}|f(x)| r d x\right)^{1 / r} \leqq A_{r}\left(\int_{0}^{2 \pi}\left|F_{n}(x)\right| r d x\right)^{1 / r}+B_{r \omega}(2 \pi / n)$,

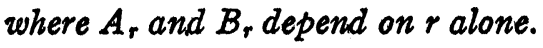

There is a trigonometric polynomial, $T_{n}(x)$, of degree $n$ such that $\left|f(x)-T_{n}(x)\right| \leqq A \omega(2 \pi / n)$, where $A$ is an absolute constant [3, p. 7]. Therefore,

$$
\begin{aligned}
\left(\int_{0}^{2 \pi}|f(x)| r d x\right)^{1 / r} \leqq & \left(\int_{0}^{2 \pi}\left|f(x)-T_{n}(x)\right| r d x\right)^{1 / r} \\
& +\left(\int_{0}^{2 \pi}\left|T_{n}(x)\right|^{r} d x\right)^{1 / r} \\
& \leqq A_{r} \omega(2 \pi / n)+\left(\int_{0}^{2 \pi}\left|T_{n}(x)\right| r d x\right)^{1 / r} .
\end{aligned}
$$

Theorem 1 of MV states that

$$
\left(\int_{0}^{2 \pi}\left|T_{n}(x)\right| r d x\right)^{1 / r} \leqq A_{r}\left(\int_{0}^{2 \pi}\left|T_{n}(x)\right| r d \phi_{2 n+1}(x)\right)^{1 / r} .
$$

In turn,

(5)

$$
\begin{aligned}
& \left(\int_{0}^{2 \pi}\left|T_{n}(x)\right| r d \phi_{2 n+1}(x)\right)^{1 / r} \\
& \leqq \\
& \quad\left(\int_{0}^{2 \pi}\left|T_{n}(x)-f(x)\right| r d \phi_{2 n+1}(x)\right)^{1 / r} \\
& \quad+\left(\int_{0}^{2 \pi}|f(x)| r d \phi_{2 n+1}(x)\right)^{1 / r} \\
& \leqq A_{r} \omega(2 \pi / n)+\left(\int_{0}^{2 \pi}|f(x)| r d \phi_{2 n+1}(x)\right)^{1 / r} .
\end{aligned}
$$

The hypothesis of dominance at the points $x_{i}{ }^{n}$, together with Theorem 2 of MV, implies that

$$
\begin{aligned}
& \left(\int_{0}^{2 \pi}|f(x)| r d \phi_{2 n+1}(x)\right)^{1 / r} \\
& \leqq\left(\int_{0}^{2 \pi}\left|F_{n}(x)\right| r d \phi_{2 n+1}(x)\right)^{1 / r} \leqq A_{r}\left(\int_{0}^{2 \pi}\left|F_{n}(x)\right| r d x\right)^{1 / r} .
\end{aligned}
$$

The combination of (3) through (6) completes the demonstration of (2). 
If $f(x)$ is a trigonometric polynomial of degree $n$, the approximation process is unnecessary and the terms in $\omega(2 \pi / n)$ do not occur.

COROLlaRy 1. If $f(x)$ is a trigonometric polynomial of degree $n$, then for $1<r<\infty$

$$
\left(\int_{0}^{2 \pi}|f(x)| r d x\right)^{1 / r} \leqq A_{r}\left(\int_{0}^{2 \pi}\left|F_{n}(x)\right| r d x\right)^{1 / r},
$$

where $A_{r}$ depends on $r$ alone.

THEOREM 2. For $0<\mu<1$,

$$
\left(\int_{0}^{2 \pi}|f(x)|^{\mu} d x\right)^{1 / \mu} \leqq A_{\mu} \int_{0}^{2 \pi}\left|F_{n}(x)\right| d x+B_{\mu} \omega(2 \pi / n),
$$

where $A_{\mu}$ and $B_{\mu}$ depend on $\mu$ alone.

Let $T_{n}(x)$ have the same connotation as above. Then by Theorem 6 of $\mathrm{MV}$,

$$
\begin{aligned}
\left(\int_{0}^{2 \pi}|f(x)|^{\mu} d x\right. & \leqq \int_{0}^{2 \pi}\left|f(x)-T_{n}(x)\right|^{\mu} d x+\int_{0}^{2 \pi}\left|T_{n}(x)\right|^{\mu} d x \\
& \leqq A_{\mu}[\omega(2 \pi / n)]^{\mu}+B_{\mu}\left(\int_{0}^{2 \pi}\left|T_{n}(x)\right| d \phi_{2 n+1}(x)\right)^{\mu} .
\end{aligned}
$$

Theorem 1 of $\mathrm{MV}$ and the hypothesis of dominance show that

$$
\begin{aligned}
\left(\int_{0}^{2 \pi}\left|T_{n}(x)\right| d \phi_{2 n+1}(x)\right)^{\mu} & \leqq \\
\leqq & \left(\int_{0}^{2 \pi}\left|T_{n}(x)-f(x)\right| d \phi_{2 n+1}(x)\right)^{\mu} \\
& +\left(\int_{0}^{2 \pi}|f(x)| d \phi_{2 n+1}(x)\right)^{\mu} \\
\leqq & A_{\mu}[\omega(2 \pi / n)]^{\mu}+\left(\int_{0}^{2 \pi}\left|F_{n}(x)\right| d \phi_{2 n+1}(x)\right)^{\mu} \\
\leqq & A_{\mu}[\omega(2 \pi / n)]^{\mu}+B_{\mu}\left(\int_{0}^{2 \pi}\left|F_{n}(x)\right| d x\right)^{\mu} .
\end{aligned}
$$

Inequalities (8) and (9) therefore imply that

$$
\int_{0}^{2 \pi}|f(x)|^{\mu} d x \leqq A_{\mu}\left(\int_{0}^{2 \pi}\left|F_{n}(x)\right| d x\right)^{\mu}+B_{\mu}[\omega(2 \pi / n)]^{\mu} .
$$


The extraction of the appropriate root and the corresponding relabelling of constants then gives (7).

Theorem 3. If $|f(x)| \leqq M$, then

$$
\begin{aligned}
\int_{0}^{2 \pi}|f(x)| d x \leqq A \log ^{+} M \int_{0}^{2 \pi}\left|F_{n}(x)\right| d x+B \\
+C \omega(2 \pi / n)\left[1+M+\log ^{+} M+C \omega(2 \pi / n)\right],
\end{aligned}
$$

where $A, B$, and $C$ are absolute constants.

Again, let $T_{n}(x)$ have the connotation as above. Then by Theorem 5 of $\mathrm{MV}$,

$$
\begin{aligned}
& \int_{0}^{2 \pi}|f(x)| d x \\
& \leqq \int_{0}^{2 \pi}\left|f(x)-T_{n}(x)\right| d x+\int_{0}^{2 \pi}\left|T_{n}(x)\right| d x \\
& \leqq A \omega(2 \pi / n)+B \int_{0}^{2 \pi}\left|T_{n}(x)\right| \log ^{+}\left|T_{n}(x)\right| d \phi_{2 n+1}(x)+C \\
& \leqq B \int_{0}^{2 \pi}|| T_{n}(x)\left|\log ^{+}\right| T_{n}(x)|-| f(x)\left|\log ^{+}\right| f(x)|| d \phi_{2 n+1}(x) \\
& \quad+A \omega(2 \pi / n)+B \int_{0}^{2 \pi}|f(x)| \log ^{+}|f(x)| d \phi_{2 n+1}(x)+C \\
& =S_{1}+A \omega(2 \pi / n)+S_{2}+C .
\end{aligned}
$$

The application of Theorem 1 of MV shows that

$$
\begin{aligned}
s_{2} & \leqq B \log ^{+} M \int_{0}^{2 \pi}|f(x)| d \phi_{2 n+1} \\
& \leqq B \log ^{+} M \int_{0}^{2 \pi}\left|F_{n}(x)\right| d \phi_{2 n+1}(x) \\
& \leqq B \log ^{+} M \int_{0}^{2 \pi}\left|F_{n}(x)\right| d x .
\end{aligned}
$$

To obtain an estimate for $S_{1}$, first suppose that for a particular value of $j$ either $\left|T_{n}\left(x_{j}{ }^{n}\right)\right| \leqq 1$ or $\left|f\left(x_{j}{ }^{n}\right)\right| \leqq 1$. If the former holds, then $\left|f\left(x_{i}^{n}\right)\right| \leqq 1+A \omega(2 \pi / n)$, and $\left|f\left(x_{i}^{n}\right)\right| \log ^{+}\left|f\left(x_{i}^{n}\right)\right| \leqq M A \omega(2 \pi / n)$. If the latter holds, then $\left|T_{n}\left(x_{j}^{n}\right)\right| \leqq \min [M+A \omega(2 \pi / n), 1+A \omega(2 \pi / n)]$, so that $\left|T_{n}\left(x_{i}^{n}\right)\right| \log ^{+}\left|T_{n}\left(x_{i}^{n}\right)\right| \leqq[M+A \omega(2 \pi / n)] A \omega(2 \pi / n)$. 
Suppose next that for a particular value of $j$, both $\left|T_{n}\left(x^{n}\right)\right|>1$ and $\left|f\left(x_{j}^{n}\right)\right|>1$. For brevity suppose also that $\left|T_{n}\left(x_{i}^{n}\right)\right| \geqq f\left(x_{j}^{n}\right)$. The other case will lead to the same conclusion. Then

$$
\begin{aligned}
\left|T_{n}\left(x_{i}^{n}\right)\right| & \left|\log ^{+}\right| T_{n}\left(x_{j}^{n}\right)|-| f\left(x_{j}^{n}\right)\left|\log ^{+}\right| f\left(x_{j}^{n}\right) \mid \\
= & \left|T_{n}\left(x_{j}^{n}\right)\right|\left[\log ^{+}\left|T_{n}\left(x_{j}^{n}\right)\right|-\log ^{+}\left|f\left(x_{j}^{n}\right)\right|\right] \\
& \left.+\log ^{+}\left|f\left(x_{j}^{n}\right)\right||| T_{n}\left(x_{j}^{n}\right)|-| f\left(x_{j}^{n}\right) \mid\right] \\
\leqq & \left|T_{n}\left(x_{j}^{n}\right)\right| \log \left[1+\left|T_{n}\left(x_{j}^{n}\right)\right|-\left|f\left(x_{j}^{n}\right)\right|\right]+A \omega(2 \pi / n) \log ^{+} M \\
\leqq & {[M+A \omega(2 \pi / n)] A \omega(2 \pi / n)+A \omega(2 \pi / n) \log ^{+} M . }
\end{aligned}
$$

In any event, the individual terms comprising the mean in $S_{1}$ are less than $A \omega(2 \pi / n)\left[M+\log ^{+} M+A \omega(2 \pi / n)\right]$, and therefore $S_{1}$ has the same bound. The theorem then follows from (10) and (11).

\section{REFERENCES}

1. P. Civin, Fourier coefficients of dominant functions, Duke Math. J. vol. 13 (1946) pp. 1-7.

2. G. H. Hardy and J. E. Littlewood, Notes on the theory of series (XIX): $A$ problem concerning majorants of Fourier series, Quart. J. Math. Oxford Ser. vol. 6 (1935) pp. 304-315.

3. D. Jackson, The theory of approximation, Amer. Math. Soc. Colloquium Publications, vol. 11, New York, 1930.

4. J. Marcinkiewicz and A. Zygmund, Mean values of trigonometric polynomials, Fund. Math. vol. 28 (1937) pp. 131-166.

UNIVERSTTY of Buffalo 